\title{
PROCESS AND SYSTEMS A Canadian Rural Living Lab Hospital: Implementing solutions for improving rural emergency care
}

\author{
Author: Richard Fleet ${ }^{\mathrm{A}}$
}

\section{Introduction}

More than 6 million Canadians live in rural areas (approximately $20 \%$ of the population) and emergency services are a critical safety net for them.

\section{Objectives}

We want to create, in Baie-Saint-Paul (rural emergency department, Québec, Canada), an experimental milieu where all stakeholders develop, implement and evaluate solutions to address the problems that beset their environment.

\section{Method}

The Living Lab will rely on the quadruple aim approach to improve health system performance and will use a multimethod approach based on the philosophy of open and user-driven innovation. Three pilot projects will be implemented (quality of work life programme, computed tomography implementation study and telemedicine in ambulances). Other possible solutions will be evaluated and prioritised (in situ simulation, care protocol, telemedicine, point-of-care ultrasound, helicopters and drones).

\section{Conclusion}

We are confident that this Living Lab will contribute to saving lives, will improve the quality of work life for rural healthcare professionals, and will inspire similar innovation internationally.

KEYWORDS: Emergency medicine, rural, Canada, Living Lab

If there is two-tiered medicine in Canada, it's not rich and poor, it's urban versus rural.

Dr John Wootton

Author: A emergency medicine physician, psychologist and associate professor, Laval University, Québec, Canada, endowed research chair of emergency medicine, Centre de recherche du CISSS Chaudière-Appalaches, Lévis, Canada and Centre de recherche sur les soins et services de première ligne de l'Université Laval, Québec, Canada

\section{Preface: Destiny and a promise to improve rural emergency care}

In the early 2000s, governments across Canada made massive healthcare cuts in rural areas, closing hospitals and reducing support services. ${ }^{2,3}$ In the rural hospital where I started my medical career in Nelson, British Columbia, the general surgery programme, intensive care and inpatient mental health unit closed; radiography as well as laboratory services were cut and there was no computed tomography (CT) scanner. In the context of geographic isolation and limited capacity for medical evacuation (med-evac), community members and healthcare professionals staunchly advocated to save their services. ${ }^{4}$ Trained like most physicians in an urban academic setting, I was not prepared. I still have chills when I think of the last patient I treated in Nelson:

At 4am, an elderly patient presented with abdominal pain. I performed bedside ultrasonography in search of an explanation for her pain. She had a large $(6.5 \mathrm{~cm})$ abdominal aortic aneurysm and urgently needed vascular surgery - only the service was $400 \mathrm{~km}$ away. While I explained the situation to her, she interrupted, 'Doctor, by the way, I want to thank you for standing up for us. I have read you and your colleagues' articles in the newspapers about your opposition to the service cuts. We used to have a fabulous hospital here before all the cuts. I understand some of you may leave, and I don't blame you.' Surprised and touched, I forged ahead with my explanation of her medical condition and arranged an urgent transfer. At the end of my shift (around 7am), she told me unequivocally, 'Doctor, if I don't see you again, promise me one thing: please continue the fight.' Unfortunately, her transfer did not go well. The plane was delayed several times, and she eventually was transferred by road ambulance - a 5-hour transport. She arrived unacceptably late, at about $6 \mathrm{pm}$. She died before surgery. ${ }^{4}$

Since 2010, our entire research programme has been inspired by this frontline clinical experience as a rural doctor. As we enter the next stage of our research, we recall that the journey started in one small rural hospital with immediate and desperate needs. We have decided to come full circle and attempt to bring about change in one small rural hospital. We want to develop the most scalable model for optimal rural care - a model that can then inspire others and effect change locally and globally. 


\section{Introduction}

More than 6 million Canadians live in rural areas (approximately $20 \%$ of the population) and emergency services are a critical safety net for these communities. ${ }^{5,6}$ Rural emergency departments (EDs) are of high importance for Canadians ( 4 million annual visits to over 300 rural EDs) and yet are frequently poorly resourced most have no access to on-site CT or surgical services. ${ }^{7-9}$ The rural population is older and in poorer health than in urban settings and are at higher risk of death. ${ }^{7-9}$ Our studies have shown that Canadian patients treated in rural hospitals are $20 \%$ more likely to die from a stroke than patients treated in urban hospitals and that rural Quebec trauma patients had pre-hospital and ED mortality rates 3.4 times higher than their urban counterparts..$^{10,11}$

We have also previously undertaken a number of research projects aimed at integrating scientific knowledge about rural EDs with the experiential knowledge of various stakeholders. ${ }^{12-14}$ Among them, the Rural Emergency Care 360 project aimed to engage rural community members, healthcare providers, managers and policymakers in identifying needs, and imagining and taking ownership of initiatives likely to improve care and services.

This project gave rise to the idea of establishing a 'Living Lab': an experimental milieu where patients, community representatives, decision makers, researchers, healthcare professionals and other stakeholders collaborate to address the persistent problems that beset their own rural environment. ${ }^{15}$

\section{Goal and objectives}

The goal is to design and implement a rural Living Lab in the ED at Baie-Saint-Paul hospital (Québec, Canada) where transformative, user-designed, point-of-care solutions will be co-developed, implemented and evaluated in a sustainable and scalable way.

We will proceed with pilot experimentation projects to bring together stakeholders, build confidence and trust, foster a culture of co-creation and demonstrate the first results to facilitate funding. Three projects emerged, on the basis of the most urgent needs as identified by local stakeholders, and will be implemented in the months to come: a work environment improvement project (Project 1a), an evaluation of the implementation of CT (Project 1b) and a telemedicine project (Project 1c).

We will then prioritise further user-designed solutions and, if selected, implement additional solutions in the Living Lab.

\section{A Living Lab with a quadruple aim approach to improve health system performance}

\section{The idea: Living Labs}

The Living Lab concept emerged from the Massachusetts Institute of Technology Media Lab at the end of 1990 and was further developed by the European Network of Living Labs (ENoLL) founded in 2006. It is a multimethod approach based on the philosophy of 'open' and 'user-driven' innovation, where experimentation takes place in real-life contexts and seeks to address real-world problems. ${ }^{15}$ Living Labs were originally conceived to meet innovation challenges faced by information and communication technology. Now, examples of Living Labs can be found in various fields including urbanism, education, technology development, and healthcare. ${ }^{15-20}$ The Living Lab process is based on a four-step iterative process involving an interdisciplinary, multi-stakeholder team. ${ }^{15}$
> Co-creation: supports the mindset of using multiple points of view to work through the challenges, constraints and opportunities that arise while creating new scenarios and concepts.

> Exploration: engages all stakeholders in the materialisation of the scenarios in a safe space where all the stakeholders can think of creative ways to solve the problems.

> Experimentation: implements scenarios with large groups of users in order to collect data that will be analysed during evaluation.

> Evaluation: assesses new ideas and innovative concepts in real-life situations through various dimensions (social, economic, cultural, patient experience, quality of care etc) and makes observations on the potential and adoption through a confrontation with users' value models.

In establishing a patient/community-centred Living Lab where evidence-based innovations to improve rural emergency care can be rapidly deployed, tested and spread, our programme seeks to optimise health system performance in the pursuit of the quadruple aim. ${ }^{21}$ Hence, the Living Lab aims to:

> improve the health and safety of the rural population. By creating a space where innovations in rural emergency medicine can be implemented and tested, the Living Labs will contribute to evidence-based healthcare and, ultimately, to improving the health of the vulnerable rural population.

> improve the quality and experience of rural emergency care for patients and their families. Our research projects, past and ongoing, invite patients and other rural emergency stakeholders to engage in addressing rural healthcare problems and providing solutions based on their needs and experiences. Dubé et al speak of the Living Lab approach as being 'user-driven'. ${ }^{16}$ This programme thus includes the implementation of user-prioritised solutions. Patients and communities are already privileged partners of the Living Lab project and its structure will reflect this. Our programme will also provide patients and other stakeholders with a better equipped ED, improving access to care locally (eg providing CT and telemedicine remote support, point-of-care ultrasound (POCUS), state-of-the-art simulation training etc).

> improve quality of work life and retention rates of rural emergency care providers. Many of the key problems identified in rural emergencies are related to human resources (recruitment, retention and qualifications). ${ }^{22,23}$ Our programme has several projects that aim to create and sustain a culture of creativity, innovation and workforce well-being, including an evidence-based wellness programme. Better equipment will also attract and retain medical specialists, and the engagement of local stakeholders in taking responsibility and ownership for solutions will reduce social and professional isolation. The initiative will attract healthcare professionals and researchers to work and establish themselves in rural regions and will reduce work absenteeism, burnout and medical errors.

> optimise per capita cost of healthcare. Our programme includes several projects (including establishing the Living Lab itself) that are based on private-public partnerships and all projects will seek value-based care. The Living Lab will help us study which solutions are cost-effective and more desirable for stakeholders. The Living Lab project will integrate the core 
principles of 'learning healthcare systems' and 'healthcarebased value'. ${ }^{24,25}$ It will lead to long-lasting collaborations between health economists, health services researchers and healthcare professionals for the design, improvement and establishment of performance measures, and help prepare for the future of healthcare.

\section{Designing and implementing the rural Living Lab Hospital in Baie-Saint-Paul}

\section{The real-life context}

Baie-Saint-Paul Hospital is a state-of-the-art facility built in 2018 to be resilient to the region's seismic potential. The ED receives an average of 13,500 visits per year. The hospital has 30 acute care beds, seven mental health beds, one ED, an intermediate critical care unit and $24 / 7$ access to basic laboratory and radiology services, general surgery and anaesthesia, as well as internal medicine and psychiatric services during the day. However, the ED is a level 3 trauma centre with no access to other medical, paediatric, obstetric and surgical specialties and no CT.

\section{Monitoring the Living Lab}

To ensure the Living Lab solutions do, in fact, improve the performance of the rural healthcare system, accountability, in keeping with the quadruple aim, needs to be built in from the start. Our data gathering approach will target the health of the rural population, patient experience of care, quality of work life and cost. We will work with Canadian databases, health ministry and agencies and local health organisation to establish meaningful databases on ED performance indicators, interfacility transfers and mortality data on emergency sensitive conditions. Chronometric and volumetric healthcare indicators will be tracked over the course of the project and years to come.

\section{Sustainability and scaling up}

The Living Lab is designed to act as a model and a reference for other rural areas that seek to improve their care. At the heart of this initiative is the idea of 'scale up' or increasing the reach and adoption of innovations. Ben Charif et al summarises the steps necessary for successful scale up: assessment of scalability (potential for scale up) of the innovation (eg effectiveness, cost-effectiveness), development of a scale up strategy, strategy implementation, strategy evaluation and assuring sustainability. ${ }^{26}$ The Living Lab governance structure will ensure that every innovation deployed goes though the steps of scale up. The scale up strategy will also be supported by the Québec, Canadian and international networks set-up around the Living Lab.

\section{Project 1: Test three user-prioritised solutions in the Living Lab}

The literature suggests that a promising way to implement a Living Lab is to proceed iteratively with pilot experimentation projects. ${ }^{15,19}$ The pilots bring together stakeholders, build confidence and trust, foster a culture of co-creation, establish mediation processes and demonstrate the first results to facilitate funding. We have already started this process; three solutions have been selected and prioritised by local stakeholders on the basis of the most urgent needs and on the strength of the environment.

Project 1a: A creative place where people want to work: improving quality of work life, preventing burnout and optimising recruitment and retention in the Living Lab

\section{Background and objectives}

The Canadian Medical Association's 2018 National Physician Survey showed alarming rates of physician burnout. ${ }^{27}$ Roughly $30 \%$ of physicians reported high levels of burnout, $32 \%$ of depression and $8 \%$ thought of ending their lives in the year previous to the survey. Rates for residents are even higher: burnout $38 \%$, depression $48 \%$ and recent suicidal ideation $15 \%$. Emergency medicine physicians and healthcare workers are at a higher risk, with several studies reporting burnout rates of up to $55 \%{ }^{28}$ A similar or worse situation has been reported for nurses at provincial, national and international levels. Rates of burnout in rural emergency care providers, however, have not yet been extensively studied. ${ }^{29}$

In this project, we will:

> assess quality of work life needs at baseline among Living Lab healthcare professionals

> introduce evidence-based stress management/wellness strategies for healthcare professionals

$>$ help design and evaluate user-driven wellness interventions to improve quality of work life in the Living Lab.

\section{Methods}

All professionals and staff from Baie-Saint-Paul involved in emergency care will be asked to participate in the project. Quality of work life and burnout levels will be measured psychometrically with '39-item rural emergency recruitment and retention questionnaire' developed by our team will help us identify work satisfaction in several domains including items on continuing education, emergency patients' access to other specialists (eg surgeons), communication quality, relationships with colleagues etc; the 'Quality of Work Life Systemic Inventory (QWLSI)' tool assesses healthcare providers' work conditions at a given time in several domains, their goals within these domains, and the priority attributed to each as well as an indication of the employees' psychological health; and 'burnout' will be measured by the validated 22 item Maslach Burnout Inventory - Health Services Survey (MBI-HSS), its three domains are emotional exhaustion, depersonalisation and personal accomplishment. ${ }^{22,30,31}$

Participants will be offered an informational session on the aspirations of the Living Lab in terms of quality of work life and an introductory intervention session on stress management strategies. Topics presented will include cognitive behavioural strategies focusing on compassion fatigue, resilience, critical incident debriefing, mindfulness, yoga and meditation, information on exercise and the mind and mood, sleep science, self-medication, substance use and misuse, the science of happiness (positive psychology), the best evidence-based recruitment and retention strategies in rural healthcare, top organisational changes for improving quality of work life, and the contribution of arts and creativity. ${ }^{32}$ Bibliotherapy (readings on subjects covered) and links to video documentaries will be included. 
A survey and focus group will evaluate the intervention and prepost analysis will be conducted on absenteeism and recruitment and retention.

\section{Impact/expected outcomes}

To the best of our knowledge, this is the first specific evidencebased wellness intervention that targets rural healthcare professionals in the Canadian healthcare system. This solution, at the heart of the Living Lab, will lead to a well-established and scalable wellness programme, reduced burnout levels and improved quality of work life, improved recruitment and retention, reduced costs related to work absenteeism, a culture of openness to innovation and creativity, and a database on quality of work life related issues.

Project 1b: Level of infrastructure and rural innovation: a case study on the implementation of CT

\section{Background and objectives}

The question of the level of diagnostic and therapeutic infrastructure required in rural areas to ensure the safety of care and a better experience for patients and professionals is at the heart of stakeholders' concerns. As the community, healthcare professionals and elected officials in Baie-Saint-Paul have been trying for more than a decade to obtain a local CT scanner and our team was asked to document this project, we chose to use it as a case study to initiate a reflection on the level of infrastructure and its role in rural innovation. Most EDs in the USA and the province of Quebec have this essential equipment to diagnose many acute conditions. ${ }^{33,34}$

The project aims to:

> describe the efforts deployed so far by healthcare providers and the community to advocate for and justify the purchase and operation of a 24/7 in-house CT scanner in Baie-Saint-Paul Hospital

$>$ perform a clinical and economic needs analysis for local CT

> conduct a pre-post study of the impact of a local CT scanner on the number of $\mathrm{CT}$ requests, time from $\mathrm{CT}$ request to exams, indications for exams, change in the inter-facility transfer patterns, critical care professionals, community, patient and decision-maker satisfaction and economic impact.

\section{Methods}

We will create a descriptive account of the efforts deployed to purchase a CT scanner (media, reports and interviews). Health economic methods will be used to examine the cost of purchasing and operating a CT scanner relative to the potential savings. Interfacility transfer analysis pre-post CT scanner installation will also be conducted.

\section{Impact/expected outcomes}

This case study will initiate a reflection on the notion of the level of infrastructure and its role in rural innovation. The results of this descriptive study will help other communities analyse their decision to purchase a CT scanner and could improve CT access to thousands of rural Canadians currently without it. The experience of mobilisation of an entire population towards the purchase of a CT scanner will bring to light many factors (financial and socio-political) underlying unequal access to healthcare.
Project 1c: Piloting and evaluating telemedicine with Reacts

\section{Background and objectives}

Inter-facility transfers, common in rural hospitals nationwide, pose many clinical and logistical challenges due to lack of medical personnel to accompany patients. From January to June 2019, 313 inter-facility transfers took place from Baie-Saint-Paul, of which 73 required an escort - most (79\%) involving a lengthy 75 -minute drive. This priority project proposes to deploy and test a telemedicine application, Reacts (Remote Education, Augmented Communication, Training and Supervision), to offer a distant medical support to nurses in place of a doctor on board of an ambulance. Reacts is a secure digital collaboration platform that enables healthcare professionals to interact dynamically at a distance through secure messaging, interactive assistance and remote procedure monitoring. Designed to meet the highest performance and safety standards, Reacts is used by many hospitals in Canada. ${ }^{35}$

The objectives of this evaluative study are to:

> describe the implementation of the technology and identify the facilitating factors and challenges related to adoption

> identify and measure the main effects of the use of technology related to:

$>$ clinical practices

$>$ the organisation of care and services

$>$ efficiency (economic value)

$>$ safety

$>$ the acceptance and satisfaction of the various stakeholders

> identify conditions for deployment, sustainability and scaling up.

\section{Methods}

In this mixed-methods case study, data will be collected mainly from existing information sources (management tracking activities and periodic reports on activities), supplemented by questionnaires and interviews on actors' perceptions and satisfaction. ${ }^{36}$ Evaluation tools will be developed according to selected indicators

\section{Impact/expected outcomes}

We will develop a rich description of the feasibility and acceptability of Reacts according to stakeholders and clinicians, identify the conditions necessary for its successful adoption and implementation, and explore its potential for sustainability and scale up in a context where many other remote EDs also seek to optimise their inter-facility transfers.

\section{The future: Prioritising, selecting and deploying other} novel solutions

For the purpose of this Living Lab, all of the possible solutions emerging from Rural Emergency Care 360 will be submitted to the Baie-Saint-Paul Living Lab stakeholders, evaluated and prioritised. We briefly present below five examples already identified by stakeholders. 
Potential solution 1: Creating a learning and teaching Living Lab: an in situ simulation training programme

\section{The challenge}

New graduates in healthcare identify lack of training and clinical exposure in rural settings as a barrier to rural practice. Rural training settings are a key factor in recruitment and retention and plays a role in patient safety. ${ }^{37,38}$ These environments also provide an attractive alternative to busy urban hospitals with too many trainees and limited time to practice technical skills under supervision. However, some suggest that rural hospitals do not provide enough exposure to resuscitation cases or severe trauma requiring procedural skills.

\section{The solution}

We will organise dedicated clinical and procedural skills rotations for emergency medicine specialty residents, College of Family Physicians of Canada (emergency medicine) residents, and for other healthcare students. Trainees as well as local healthcare providers will take part in regular simulation training sessions in a new, in situ, state-of-the-art simulation lab. At the same time, trainees will take part in the Living Lab's ongoing innovative research projects, exchange knowledge and experience with local doctors, meet rural residents and take part in community life.

Potential solution 2: The use of emergency care protocols, prioritising paediatric emergency care

\section{The challenge}

Limited exposure to complex emergency cases as well as information overload contributes to medical errors, especially under stressful practice conditions. The use of well-established treatment protocols standardises care, minimises errors and helps establish confidence and respect in rural practice. Current protocols are not uniformly implemented and updated. ${ }^{39}$ In particular, our research shows that the treatment of sick children is highly stressful and that care protocols for this is of high priority for rural care providers.

\section{The solution}

We will deploy the Transfer Emergency Knowledge for Kids (TREKK) protocols and its mobile app. These were developed by leading Canadian paediatric emergency physicians and researchers and a group of leading experts in knowledge translation. TREKK will be used on local computers and mobile devices in the Baie-Saint-Paul Hospital ED and could be beamed onto a giant screen in the resuscitation and trauma rooms.

\section{Potential solution 3: Telemedicine/teletrauma/ telecardiology/telestroke}

\section{The challenge}

Limited access to specialists, staff as well as the solo physician practice typical of rural EDs poses a challenge for the management of complex, acutely ill, shock or trauma cases in rural hospitals. Frequent, untimely inter-facility transfers occur because of the real or perceived incapacity to meet the needs of the patient. Some of these transfers could be prioritised and others avoided through telemedicine support. ${ }^{40}$

\section{The solution}

Telemedicine support will be an integral part of the Living Lab. The plan is to equip the trauma and resuscitation rooms with audiovisual equipment and link this to referral centres.

\section{Potential solution 4: Emergency ultrasound or point-of- care ultrasound}

\section{The challenge}

Limited access to formal ultrasound, CT and specialists delays timely management of critical conditions. Our study showed that $75 \%$ of rural ED physicians believed that POCUS was an essential tool and skill to master in rural EDs, but few physicians use it regularly..$^{41}$ Learning its use is difficult due to little access to continuing medical education.

\section{The solution}

POCUS is a compact and portable ultrasound for evaluating emergency medical conditions in settings such as EDs, where it is used to guide resuscitation and monitor critically ill patients, provide procedural guidance for improved safety and confirm clinical diagnosis. We will organise training and certification to the highest level for every practising physician in the ED in collaboration with the university, continuing education units and the Canadian Association of Emergency Physicians training and via the Reacts platform, which enables the integration of teleultrasound. ${ }^{42}$

\section{Potential solution 5: Solutions from the sky - helicopters} and drones

\section{The challenge}

Québec does not have a national helicopter system. In emergency sensitive cases, such as trauma, time is of the essence. Our recent trauma study showed that mortality in rural settings was 3.4 times higher than in urban environments and that $50 \%$ of trauma deaths occurred in the pre-hospital setting. ${ }^{11}$ Locating victims and preparing timely trauma response and transport is critical. Yet in rural and remote areas it can be difficult to locate victims and deliver the necessary medical equipment (defibrillators and blood products) in a timely manner.

\section{The solution}

Our ongoing Delphi trauma study and rural summit suggest a helicopter med-evac is of high priority. A national private-public helicopter med-evac pilot programme is currently being discussed with the Québec Ministry of Health. Regarding victim location and equipment delivery, the integration of drones into the pre-hospital system could save lives through their ability to transport blood products and defibrillators in emergency situations. ${ }^{42}$

\section{Conclusion}

We are confident that the Baie-Saint-Paul Hospital Living Lab will contribute to saving lives, will improve the quality of work life for rural healthcare professionals, and will inspire similar innovation internationally. To the best of our knowledge, this will constitute the first ever establishment of a rural Living Lab hospital in Canada. We expect that our inclusive, patient and communitycentred local model of governance will help attain the quadruple aims of healthcare performance as it evolves. With our large and 
experienced interdisciplinary team of investigators, national and international observers, patients and community stakeholders, professional associations and local and provincial government members, we expect that this project will be a success and that these successes will be replicated elsewhere. An undertaking like this is necessarily iterative: both successes and failures will be duly noted and learned from.

This research programme, which is firmly committed to access and quality of care for rural populations, will, I hope, enable me to honour the promise I made as a doctor to my patient from Nelson who asked me to continue fighting for rural populations. By proposing this 'total' project for a rural emergency and envisioning it from the outset as a model to follow and imitate, I hope to enact the truth of this quote, taken from an interview with a doctor conducted as part of the Rural Emergency Care 360 project:

We always have the impression that innovations are made in the tertiary centre; that's where the research is cutting-edge and all. But precisely because ... it is a little less dogmatic, there are many things here that we do ... that are almost more innovative than what I have seen in the city.

\section{Acknowledgments}

I would like to thank Dr Louella Vaughan, senior clinical fellow at Nuffield Trust, one of our international collaborators in this Living Lab project, for her advice on this manuscript and for giving us the opportunity to present some of the solutions for rural emergency care proposed in this manuscript to the Nuffield Trust and partners in London in May 2018.

I would also like to thank France Légaré, Mylaine Breton, JeanPierre Després, Julien Poitras, Isabelle Gaboury, Jean-Paul Fortin, Jean-Frédéric Lévesques, Kim Lavoie, Gilles Dupuis, Jean-Sébastien Marchand, Hassane Alami, Fatoumata Tounkara, Alison Coates, Julie Théberge, Mélanie-Ann Smithmann, Mélissa McDonald, Catherine Turgeon-Pelchat and Louisa Blair for helpful comments on a preliminary version of this article.

\section{References}

1 Wooton J. New office to focus on rural health issues. Farm Family Health 1999;7.

2 Larsen Soles TM. A strategic plan for eliminating rural hospital services through the process of regionalization. Can J Rural Med 2005;10:107-8.

3 Regan S, Wong ST. Patient perspectives on primary health care in rural communities: effects of geography on access, continuity and efficiency. Rural Remote Health 2009;9:1142.

4 Fleet R, Plant J, Ness R, Moola S. Patient advocacy by rural emergency physicians after major service cuts: the case of Nelson, BC. Can J Rural Med 2013;18:56-61.

5 Statistics Canada. Number of persons in the total population and the farm population, for rural areas and population centres classified by sex and age: Table 32-10-0012-01. Statistics Canada, 2016.

6 Haggerty JL, Roberge D, Pineault R, Larouche D, Touati N. Features of primary healthcare clinics associated with patients' utilization of emergency rooms: urban-rural differences. Healthcare Policy 2007:3:72.

7 DesMeules M, Pong R, Lagacé $\mathrm{C}$ et al. How healthy are rural Canadians? An assessment of their health status and health determinants. Ottawa: Canadian Institute for Health Information, 2006.

8 Statistics Canada. Leading causes of death, total population, by age group: Table 13-10-0394-01. Statistics Canada, 2016.
9 Peek-Asa C, Zwerling C, Stallones L. Acute traumatic injuries in rural populations. Am J Public Health 2004;94:1689-93.

10 Fleet R, Bussières S, Tounkara FK et al. Rural versus urban academic hospital mortality following stroke in Canada. PLoS One 2018;13:e0191151.

11 Fleet R, Lauzier F, Tounkara FK et al. Profile of trauma mortality and trauma care resources at rural emergency departments and urban trauma centres in Quebec: a population-based, retrospective cohort study. BMJ Open 2019;9:e028512.

12 Fleet R, Tounkara FK, Ouimet M et al. Portrait of trauma care in Quebec's rural emergency departments and identification of priority intervention needs to improve the quality of care: a study protocol. BMJ Open 2016;6:e010900.

13 Fleet R, Dupuis G, Fortin J-P et al. Rural emergency care $360^{\circ}$ : mobilising healthcare professionals, decision-makers, patients and citizens to improve rural emergency care in the province of Quebec, Canada: a qualitative study protocol. BMJ Open 2017;7:e016039.

14 Fleet R, Turgeon-Pelchat C, Trottier J-G et al. Organisation des services dans une urgence rurale eloignee: reflexions autour du cas de Fermont, Quebec. Can J Rural Med 2018;23:106-12.

15 Dubé P, Sarrailh J, Billebaud C et al. Qu'est-ce qu'un Living Lab? Le livre blanc des Linving Labs. Montreal: Umvelt, 2014:133.

16 Følstad A. Living labs for innovation and development of information and communication technology: a literature review. Electronic J Organizational Virtualness 2008;2:99-131.

17 French M, Miller FA. Leveraging the 'living laboratory': on the emergence of the entrepreneurial hospital. Soc Sci Med 2012;75:717-24.

18 Bergvall-Kåreborn B, Ståhlbröst A. Living Lab: an open and citizencentric approach for innovation. Int J Innovation Regional Dev 2009;1:356-70.

19 Veeckman C, Schuurman D, Leminen S, Westerlund M. Linking living lab characteristics and their outcomes: Towards a conceptual framework. Technol Innovation Manag Rev 2013;3:6-15.

20 Spinelli G, Weaver P, Marks M, Victor C. Making a case for creating living labs for aging-in-place: enabling socially innovative models for experimentation and complementary economies. Front Sociol 2019;4:19.

21 Bodenheimer T, Sinsky C. From triple to quadruple aim: care of the patient requires care of the provider. Ann Fam Med 2014;12: 573-6.

22 Bragard I, Fleet R, Etienne AM et al. Quality of work life of rural emergency department nurses and physicians: a pilot study. BMC Res Notes 2015;8:116.

23 Kabene SM, Orchard C, Howard JM, Soriano MA, Leduc R. The importance of human resources management in health care: a global context. Hum Resour Health 2006;4:20.

24 Friedman CP, Wong AK, Blumenthal D. Achieving a nationwide learning health system. Sci Transl Med 2010;2:57cm29.

25 Porter ME. What is value in health care? N Engl J Med 2010; 363:2477-81.

26 Ben Charif A, Hassani K, Wong ST et al. Assessment of scalability of evidence-based innovations in community-based primary health care: a cross-sectional study. CMAJ Open 2018;6:E520-7.

27 Canadian Medical Association. CMA national physician health survey: A national snapshot. CMA, 2018.

28 Arora M, Asha S, Chinnappa J, Diwan AD. Review article: Burnout in emergency medicine physicians. Emerg Med Australas 2013; 25:491-5.

29 The Lancet. Physician burnout: a global crisis. Lancet 2019;394:93.

30 Martel JP, Dupuis G. Quality of work life: theoretical and methodological problems, and presentation of a new model and measuring. Soc Indic Res 2006;77:333-68.

31 Schaufeli WB. Past performance and future perspectives of burnout research. SA J Industrial Psychol 2003;29:1-15.

32 Lambert PD (ed). Managing arts programs in healthcare. Routledge, 2016.

33 Bergeron C, Fleet R, Tounkara FK, Lavallée-Bourget I, TurgeonPelchat C. Lack of CT scanner in a rural emergency department 
increases inter-facility transfers: a pilot study. BMC Res Notes 2017; 10:772.

34 Joynt KE, Harris Y, Orav EJ, Jha AK. Quality of care and patient outcomes in critical access rural hospitals. JAMA 2011;306:45-52.

35 Service de l'évaluation des technologies et des modes d'intervention en santé du Centre hospitalier de l'Université de Montréal. Expérimentation de Reacts comme outil de téléassistance en neurochirurgie. CHUM, 2015

36 Yin RK. Case study research and applications: Design and methods. Sage publications, 2017.

37 Curran V, Rourke J. The role of medical education in the recruitment and retention of rural physicians. Med Teach 2004;26:265-72.

38 Aggarwal R, Mytton OT, Derbrew $M$ et al. Training and simulation for patient safety. BMJ Qual Saf 2010;19(Suppl 2):i34-43.

39 Ebben $\mathrm{RH}$, Vloet LC, Verhofstad MH et al. Adherence to guidelines and protocols in the prehospital and emergency care setting: a systematic review. Scand J Trauma Resusc Emerg Med 2013;21:9.
40 Duchesne JC, Kyle A, Simmons ] et al. Impact of telemedicine upon rural trauma care. J Trauma 2008:64:92-8.

41 Leger P, Fleet R, Maltais-Giguere ] et al. A majority of rural emergency departments in the province of Quebec use point-of-care ultrasound: a cross-sectional survey. BMC Emerg Med 2015;15:36.

$42 \mathrm{Kim} \mathrm{SJ}$, Lim G], Cho J, Côté MJ. Drone-aided healthcare services for patients with chronic diseases in rural areas. J Intelligent Robotic Syst 2017;88:163-80.

Address for correspondence: Dr Richard Fleet, Bureau 4617, Pavillon Ferdinand-Vandry, 1050 Avenue de la Médecine, Québec (QC) G1V 0A6, Canada.

Email: richard.fleet@fmed.ulaval.ca

\section{Royal College of Physicians}

\section{Physicians and global health}

The final volume of the RCP500 book series explores the influence that members and fellows of the RCP have had on global health - from the college's 16th-century beginnings to the present day - with doctors describing their experiences in fields ranging from diving medicine in Australia to researching altitude sickness in South America.

\section{Order by phone or online Tel +44(0)2030751358 or visit www.rcplondon.ac.uk/shop}

Price: $£ 12$ (Postage and packaging not included) $10 \%$ discount for fellows and members, $15 \%$ discount for foundation doctors and students

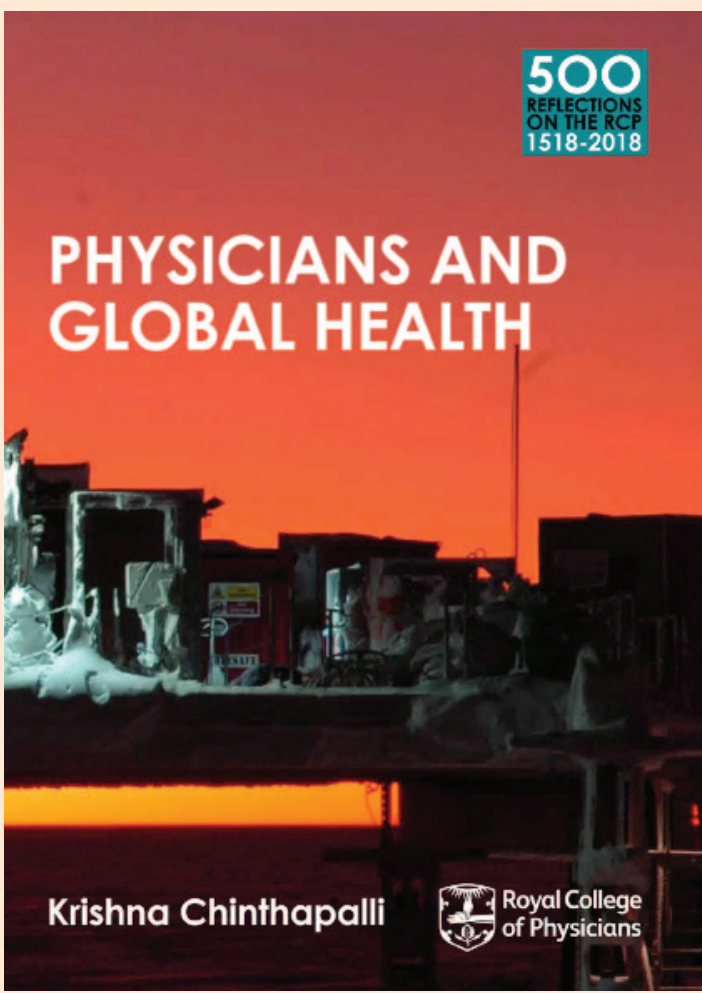

